\title{
A Network-Based Impact Measure for Propagated Losses in a Supply Chain Network Consisting of Resilient Components
}

\author{
Jesus Felix Bayta Valenzuela $\left(\mathbb{D},{ }^{1}\right.$ Xiuju Fu, ${ }^{1}$ Gaoxi Xiao, ${ }^{2}$ and Rick Siow Mong Goh ${ }^{1}$ \\ ${ }^{1}$ Computing Science Department, Institute of High Performance Computing, Agency for Science, Technology and Research, \\ 1 Fusionopolis Way, 16-16 Connexis North, Singapore 138632 \\ ${ }^{2}$ School of Electrical and Electronic Engineering, College of Engineering, Nanyang Technological University, Block S2.1, \\ 50 Nanyang Avenue, Singapore 639798
}

Correspondence should be addressed to Jesus Felix Bayta Valenzuela; jesus-valenzuela@ihpc.a-star.edu.sg

Received 3 May 2017; Accepted 1 January 2018; Published 19 February 2018

Academic Editor: Pietro De Lellis

Copyright (C) 2018 Jesus Felix Bayta Valenzuela et al. This is an open access article distributed under the Creative Commons Attribution License, which permits unrestricted use, distribution, and reproduction in any medium, provided the original work is properly cited.

\begin{abstract}
The topology of a supply chain network affects the impacts of disruptions in it. We formulate a network-based measure of the impact of a disruption loss in a supply chain propagating downstream from an originating node. The measure takes into account the loss profile of the originating node, the structure of the supply network, and the resilience of the network components. We obtain an analytical expression for the impact measure under a beta-distributed initial loss (generalizable to any continuous distribution supported on the interval $[0,1]$ ), under a breakthrough scenario (in which a fraction of the initial production loss reaches a focal company downstream as opposed to containment upstream or at the originating point). Furthermore, we obtain a closed-form solution for a supply chain network with a $k$-ary tree topology; a numerical study is performed for a scale-free network and a random network. Our proposed approach enables the evaluation of potential losses for a focal company considering its supply chain network structure, which may help the company to plan or redesign a robust and resilient network in response to different types of disruptions.
\end{abstract}

\section{Introduction}

In supply chain networks, the mitigation of production losses arising from upstream disruptions is an important aspect of their management. Another important aspect is identifying which components of the network are bound to play a key role in either spreading or mitigating such an impact, based on their positions in the network as well as their inherent risk profiles; a component's critical position in the supply chain network may amplify the effects arising from its disruption.

The impacts of disruptions on supply chain networks can be seen in the aftermath of the floods which affected Thailand in 2011 and the earthquake and tsunami which affected the Tohoku region of Japan in the same year. Haraguchi and Lall [1] made an assessment of the impacts of the floods on both industries and the local economy, as well as overseas companies in both the automobile and the electronics sectors. Saito et al. [2] examined the impacts of the Tohoku earthquake and tsunami on the sales growth and transaction relationships of firms outside the affected areas (but have suppliers and customers within the affected areas) and found significant negative effects propagated downstream to firms as far as five degrees of separation away.

In an analysis of a survey conducted among various organizations and firms in 2017, the Business Continuity Institute reported that $65 \%$ of the respondents experienced at least one disruption within the past 12 months [3], a decrease from $70 \%$ in the previous year's survey [4]. The same series of surveys reveals patterns in the origins of disruptions in supply networks. Disruptive events arose predominantly in "Tier 1," or direct, suppliers (44\% in 2017; $41 \%$ in 2016), and "Tier 2," those suppliers' own suppliers (24\% in 2017; 17\% in 2016). Cumulative losses of at least $€ 1,000,000$ became less frequent (22\% in 2017; $34 \%$ in 2016). However, the frequency of events costing the same amount increased over the same period (23\% in 2017; $9 \%$ in 2016). 
These results illustrate the need for awareness by companies of upstream conditions and effective anticipation and compensation for disruptions arising there. This need is more and more keenly felt: respondents without originidentification mechanisms for disruptive events drastically decreased from $40 \%$ in 2016 to $22 \%$ in 2017 . As the intercomponent relationships of a supply chain can be mapped onto a network, network theory offers a natural way to help fulfill such a need. By examining how an initial loss of an entity propagates downstream in the network, the impacts of the entity's loss on the other entities in the chain can be measured.

Aspects of network theory have been applied in supply chain management, specifically in the context of analyzing the relationships between the components of the chain [5]. Network theory also provides a natural context in which risks associated with a network's components or the whole network itself can be determined: a loss sustained by a component due to some adverse event may propagate downstream in a cascade of losses, unless mechanisms to mitigate losses had been put in place. Such a process is not restricted to supply chain networks alone but can be found elsewhere, such as in banking and financial networks [6, 7], organizational networks [8], production and input-output networks [9], infrastructure networks [10], and indeed any network consisting of interconnected, vulnerable entities [11-13].

In this work, we examine a different aspect of the propagation of the impact of a disruption event in a supply network. Specifically, we look at the production losses sustained by a member of the network due to a disruption occurring elsewhere. In addition, unlike previous studies, we consider the network as composed of resilient members. In other words, the elements of the network have the capability to partially or fully cushion losses which they otherwise would have sustained. The propagation of losses is thus potentially attenuated due to the resilience of individual components. Under such a scenario we seek the conditions where an initial loss sustained by a network component succeeds in propagating, in the face of attenuation at intervening components. We situate our work in this paper in the context of social network theory-based perspectives in supply chain risk management [14], as well as under supply chain risk assessment (specifically, within generic risk assessment), under the framework proposed by Ho et al. in 2015 [15].

The rest of this work is divided as follows. We review some of the related literatures in the second section. In the third section, we consider the downstream propagation of an initial disruption (measured as a fraction of the production lost) in a directed, acyclic network from an initial node, where each node has a certain degree of resilience to the disruption. We make a simplifying approximation to the equations we obtain and show that the simplification does not incur loss of accuracy. We then combine this propagated loss with a communicability-based measure first introduced by Estrada and Hatano [16] to yield an analytic expression for the measure of impact a production loss in a node has over its downstream nodes. Afterwards, we obtain an expression for the distribution and the $n$th moment of this impact measure, assuming that the production loss fraction follows a beta distribution.
The fourth section contains calculations using the impact measure for three different network structures. We first obtain exact results for a perfect $k$-ary tree, with the assumption that the nodes in the tree are identically resilient to production loss. Afterwards, we obtained computational results for two acyclic networks: one is a scale-free network, and the other is an Erdös-Rényi network. The work is then summarized in the fifth and last section.

\section{Related Literature}

2.1. Supply Chain Risk Management Definitions and Frameworks. Consensus for the definition of "supply chain risk management (SCRM)," as well as frameworks for it, had been slow to form. This is in part due to the relative nascence of the field, the diversity of definitions among researchers and practitioners, and perspective gaps between the latter two groups. In surveys of both research teams and company executives, Sodhi et al. [17] uncovered research gaps in SCRM, ranging from a lack of clear consensus in its definition (a definition gap) to a lack of studies in response to supply chain risk incidents (a process gap) and to a shortage of empirical research (a methodology gap). Within the same work, in a review of research articles covering SCRM up to that point (with the exception of most works which used mathematical modeling as their main methodology [18]), they also found an abundance of literatures on risk identification, but a surprisingly low number of works specifically focusing on risk assessment. Other works dealt with risk assessment and mitigation on a conceptual basis, or in the context of the wider SCRM framework. Furthermore, most of the surveyed literatures on risk response only covered high-frequency, lowimpact events (operational risks).

Tang's 2006 review [18], focusing on quantitative models for managing supply chain risks, sketched out a framework for classifying the SCRM articles as dealing with supply management (including supply network design, with a focus on mixed-integer models), demand management (dealing with strategies to shift demand across time, markets, or products), product management, and information management, respectively. He then provided a discussion of robust strategies for mitigating operational and disruption risks for each of the four management subdivisions. He outlined two properties of robust strategies: efficiency, enabling a company to manage operational risks in spite of occurrence of major disruptions, and resiliency, enabling sustained operations during and quick recovery after major disruptions.

Two more recent reviews attempted to cover subsequent works and to develop frameworks for SCRM. In a review focusing on SCRM enablers, Kilubi and Haasis [14] performed a review of 80 articles from 2000 to 2015, covering definitions, research methodologies, and linkages between SCRM and performance. They found disparities in definitions of SCRM and proposed a definition of it as "the identification, assessment, monitoring and evaluation of risks and potential threats within and outside supply chain networks with all members and entities involved." They also identified 12 main enablers in the literature: visibility, flexibility, relationships, redundancy, coordination, 
postponement, multiple sourcing, collaboration, risk awareness, agility, avoidance, contingency planning, risk monitoring, and the transferring and sharing of risks. Of these, they label six (visibility, relationships, collaboration, coordination, multiple sourcing, and postponement and redundancy) as preventive enablers, aiming at reducing the probability of occurrence of risk events. Five SCRM enablers (visibility, flexibility, multiple sourcing, redundancy, and coordination) were labeled as responsive enablers, focusing on mitigating adverse effects of risk events. Visibility, multiple sourcing, and redundancy are labeled both preventive and responsive and thus are of central importance among enablers. Lastly, they point to incorporating insights from social network theory as an avenue for future research. Some work on this front had been done by Hearnshaw and Wilson [19], who proposed that a supply chain network is efficient if it has a scale-free structure (a short characteristic path length, a high clustering coefficient, and a power-law degree distribution). Here, "efficiency" is from a focal company's point of view: its supply chain is efficient if it can rapidly fulfill a customer's product order even in the absence of stockpiles.

Ho et al. [15] sought to bring together insights from a large corpus of recent articles (224 articles between 2003 and 2013), for the purposes of classification, identification of recent developments, and exploration of potential research gaps. In doing so, they proposed new definitions for supply chain risk ("the likelihood and impact of unexpected macro and/or micro level events or conditions that adversely influence any part of a supply chain leading to operational, tactical, or strategic level failures or irregularities") and SCRM ("an interorganisational collaborative endeavour utilizing quantitative and qualitative risk management methodologies to identify, evaluate, mitigate, and monitor unexpected macro and micro level events or conditions, which might adversely impact any part of a supply chain"). Reviewed articles were classified into the SCRM processes they cover: risk identification, assessment, mitigation, and monitoring. Identificationfocused papers focused on developing methods for identifying potential risks, however without strong efforts on evaluating the impacts of such risks. Assessment-oriented papers focused on quantifying the impacts of various risk types (e.g., macrorisk, demand risk, manufacturing risk, supply risk, transportation risk, financial risk, and information risk) as well as generic risk assessment. They also include risk modeling, assessments of the relationships between supply chain risk and strategies, and evaluations of supply chain resilience. Their classification of risk mitigation articles follows the same schema as for risk assessment articles, using the same risk subtypes. The articles reviewed employed various modeling techniques, from linear programming models to simulations. In contrast, however, risk monitoring seems to have received less attention, with fewer articles classified as pertaining to it.

Somewhat different from the previous articles, a review by Olson and Swenseth [20] examined supply chain risk from a systems-theoretic perspective, involving tradeoffs in balancing costs, risk, and, increasingly, environmental considerations, for green supply chain management, risk, and efficiency. The authors discussed the usefulness of applying systems thinking to the decision-making process in supply chain management, to more effectively handle these tradeoffs.

2.2. Systemic Vulnerabilities, Disruptions, and Propagation in Networks. Network approaches are a natural fit to the examination of multiple interconnected and interacting agents, both as individuals and as a whole. Thus, they provide a natural framework for considering systemic risks and propagation of shock events.

Acemoglu et al. [11] proposed a framework for the study of propagating microeconomic shocks via network interactions and how these shocks translate to macroeconomic ones. Their framework also enables the characterization of an economy's macrostate performance stemming from its characteristic network interactions, as well as identification of the key network actors using centrality considerations.

Another treatment of propagating, or cascading, shocks, or failures in interdependent networks was made by Buldyrev et al. [12] in 2010. The authors developed a framework for evaluating the robustness of interacting networks in the face of cascading failures. With this, they demonstrated the presence of a critical fraction of network nodes (agents), upon whose failures will cause network fragmentation into disconnected components. Contrary to the established results for single networks (robust to failures of random nodes but susceptible to targeted disruptions), they show that the opposite holds true for interdependent networks (more vulnerable to random failures than systematic disruptions).

Systemic risks in banking networks were examined by Haldane and May [6] in a 2011 article. Drawing inspiration from ecological and epidemic networks, they identify possible mechanisms by which the initial shock caused by a single bank's collapse can propagate across the network. Firstly, a bank failure potentially leads to a collapse in turn of its creditors, causing a cascade of interbank loan-driven failures. This, however, tends to be attenuated due to losses being subdivided among a failing bank's creditors. Secondly, market liquidity shocks can generate losses in the value of a bank's external assets and potentially propagate the shock. In contrast with loan-driven failures, liquidity shocks tend to amplify with more banks failing, causing even small liquidity shocks to contribute strongly to systemic risk. A third mechanism, liquidity hoarding, can arise from hoarding of liquidity in interbank funding markets, causing a decrease in the availability of interbank loans. Liquidity hoarding can cascade through a banking network, resulting in a shock not subject to attenuation.

Laszka et al. [7] proposed a framework for estimating the systematic risk in networks. In their model, the risk of a node being compromised (or failing) may come from outside the network system ("direct compromise") or propagate to it from a neighboring node ("indirect compromise"), with direct compromise being the only propagatable risk. The authors then calculated the network's loss distribution (the probability that a given fraction of the nodes in the network becomes compromised) and the loss distribution of a subset of nodes (similar to the former distribution but restricted to a given subset of nodes in the network) in accordance with the model rules. They tested the model on two large 
real-life networks (a network of common-policy groups of IP addresses called autonomous systems participating in the Internet's routing system, and the network of Facebook users), as well as scale-free models of the two networks. As their research was made with an eye on quantifying the insurability of the components of a network, they also computed the safety loading, a measure of the expensiveness in insuring a subset of the network nodes. They showed that while predicting the risk to a network using data from a subset is very challenging due to underestimation, it is nevertheless still possible.

Shabnam et al. [8] proposed a methodology for the measurement of risk in an organizational network utilizing $i^{*}$ (an agent-oriented conceptual modeling framework) and BPMN (business process modeling notation) frameworks, combined with a simple propagation of a node's vulnerability measures to its dependencies. Blöchl et al. [9] examined input-output networks (networks of flows of goods and services between economic sectors) and defined node centrality measures (based on random walks on the network) suitable in identifying the key sectors in an economy. Using these metrics, they find commonalities in the network structures of economic networks which share geographical proximity and similar developmental status. Chopra and Khanna [10] also used the input-output network framework to examine the resilience of the United States' economy and found that it is vulnerable due to greater interdependencies of its critical infrastructure sectors (CIS).

Nagurney and Qiang [21] reviewed developments in analytical tools for the assessment of network vulnerability and robustness. In the review they showed how appropriate network measures can capture, besides network topology, underlying behavior, network flows, and induced costs. Furthermore, they proposed ways to measure the synergy associated with network integration, focusing on topological changes in supply chain networks (such as those induced by corporate mergers and acquisitions, as well as teams and partnerships for disaster relief).

More recently, Garvey et al. [22] proposed a model for the propagation of risk in a supply chain network. They made use of the structure of the supply chain and the nodes' individual risk profiles to construct a Bayesian Network (BN) of the causal risk relationships in the supply chain. They then developed risk measures based on the model and performed simulations to verify these measures. Käki et al. [23] also utilized the BN framework in modeling the propagation of risks across a supply chain network but combined it with probabilistic risk assessment (PRA) to evaluate supply network risks. A different approach was proposed by Yildiz et al. [24], where the reliability of a supply chain network (using a metric that takes into account both a network component's intrinsic reliability and the reliability of its upstream) is used as a target for optimization, along with calculated cost, in a multiobjective nonlinear programming model. This model is then solved using a novel fusion of a genetic algorithm for the network design and linear programming for the optimization of the network flow. A third approach is examined by $\mathrm{Xu}$ et al. [25], who modeled the propagation of losses in a threetier supply chain where information sharing and multiple sourcing for a focal company are present and the resulting value-at-risk (VaR).

\section{Materials and Methods}

3.1. Network Loss Propagation. Let us consider G, a directed, acyclic network (a directed, acyclic graph, DAG) of size $N$. This is a representation of, for example, the flow of materials in a supply chain network from the raw materials, through intermediate processed materials, and down to the finished product. We assume that there is a single type of flow through the network; a treatment of multiple flow types is beyond the scope of this work.

Furthermore, implicit in the acyclic formulation of a supply chain network is the assumption that the processing of a material at a given stage would not require material from downstream inputs, that is, from further processed stages of the material produced in the same supply network. This is a reasonable assumption for a wide variety of materials and products. The acyclic formulation also simplifies some of the mathematical steps in the formulation of the impact measure, as discussed below.

Given two nodes, $i$ and $j$, we call $i$ a supplier of $j$ and $j$ a customer of $i$, if a path from $i$ to $j$ exists. Node $i$ is a direct supplier of $j$ if the path is of (unweighted) length 1 (i.e., if a directed edge from $i$ to $j$ exists) and an indirect supplier otherwise. We use analogous definitions for direct and indirect customers.

The weight of the edge between any two adjacent nodes $i$ and $j$ is normalized such that the sum of all weights incident on $j$ equals 1 . Equivalently, if $\mathbf{W}$ is the (weighted) adjacency matrix of $\mathbf{G}$, then we require that $\sum_{i} W_{i j}=1$. We can think of $W_{i j}$ as the fraction of $j$ 's input provided by a direct supplier $i$. Furthermore, as $\mathbf{G}$ is acyclic, loops are excluded and downstream inputs are nonexistent. These include selfloops, edges which lead from a node to itself $\left(W_{i i}=0\right)$. This implies that nodes do not produce materials which are their own inputs but strictly receive them from upstream suppliers.

$\mathbf{W}$, the weighted adjacency matrix of $\mathbf{G}$, has an important property which we will use in subsequent sections. In $\mathbf{G}$, a path of length $L$ exists between two different nodes $i$ and $j$ if and only if $\left[\mathbf{W}^{L}\right]_{i j}$, the $i j$ th element of the $L$ th power of $\mathbf{W}$, is nonzero [26, pp. 136-137]. To see this, let us explicitly write out the sums for $L=2$ and $L=3$, as well as the general case:

$$
\begin{aligned}
{\left[\mathbf{W}^{2}\right]_{i j} } & =\sum_{k} W_{i k} W_{k j}, \\
{\left[\mathbf{W}^{3}\right]_{i j} } & =\sum_{k} \sum_{m} W_{i k} W_{k m} W_{m j} \\
{\left[\mathbf{W}^{L}\right]_{i j} } & =\underbrace{\sum_{k} \cdots \sum_{m}}_{L-1} W_{i k} \cdots W_{m j} .
\end{aligned}
$$

We see that for a path of length 2 to exist between $i$ and $j$ (i.e., two directed edges exist such that one goes from $i$ to an intermediate node and the other from the intermediate node to $j$ ), by (1), there must be at least one node $k$ for which both $W_{i k}$ and $W_{k j}$ are nonzero, as otherwise zero indicates the 


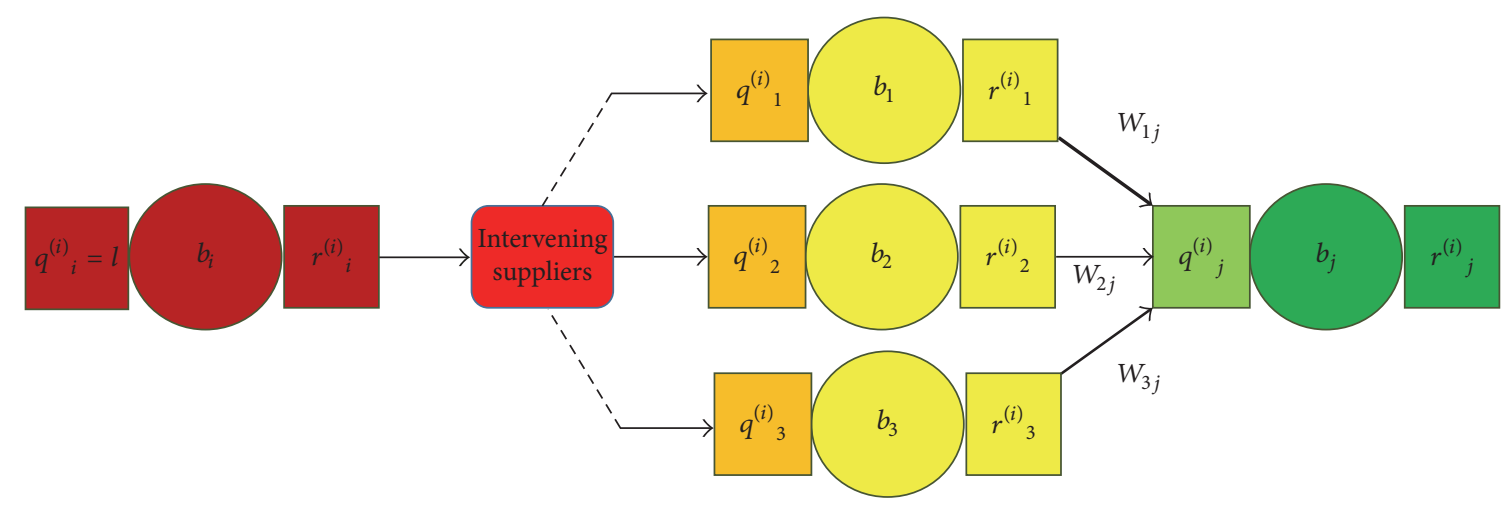

FIGURE 1: Schematic representation of a supply chain network as a directed, acyclic graph. Node $i$ represents where an initial loss $l$ originates. The loss may propagate downstream through intervening nodes (omitted) to node $j$. Also shown are three nodes representing immediate suppliers of $j$.

absence of a corresponding edge. Similarly an $L=3$ path exists between $i$ and $j$ if, by (2), at least one pair of nodes $k$ and $m$, different from $i, j$, and each other, can be found such that the three factors on the right hand side are all nonzero. For the general case of a path with length $L$ between $i$ and $j$, (3) implies that $L-1$ other distinct intermediate nodes must be present. The cases where any node index equals another correspond to self-loops, which are assumed to be absent and thus do not contribute to the sum. We further observe that the right hand side of (3) requires exactly $L$ nonzero factors, each corresponding to a directed edge along the path from $i$ to $j$.

Let us consider a scenario where node $i$ (the originating node) incurs a production loss. Depending on the properties of $i$ and intervening nodes, this loss might propagate through the network and reach node $j$ downstream, resulting in a potential loss at the latter node. In this context, we take $l \in$ $[0,1]$ as the production loss fraction sustained by $i$ due to the disruption, $q_{j}^{(i)}(l) \in[0,1]$ as the potential loss fraction of $j$ downstream from $i$ which can be sustained from a loss of $l$, and $r_{j}^{(i)}(l)$ as the actual loss fraction at $j$ due to $i$.

Each node (including $i$ and $j$ ) also has a resilience threshold, $b \in[0,1]$, which represents the extent to which a node can compensate for loss in its total inputs. A node with $b=0.5$ can compensate for up to a $50 \%$ loss in its input without its total output changing. A resilience $b$ of 1 indicates that the node can completely absorb any potential loss propagated from $i$, while a resilience of 0 means that any loss cannot be absorbed, and hence the actual loss equals the potential loss. This can represent the aggregation of the node firm's mechanisms in place in-house to compensate for input loss (such as safety stocks), which will allow it to partially or completely compensate for production losses upstream.

The loss $l$ may be propagated through or attenuated by intervening nodes, depending on their individual resilience thresholds. Let the index $k$ denote the immediate suppliers of node $j$ ( $j$ 's upstream neighbors) and $b_{j}$ be $j$ 's resilience, as previously defined. Then $q_{j}^{(i)}(l)$ is the sum of the actual loss fractions of $j$ 's immediate suppliers, $r_{k}^{(i)}(l)$, multiplied by their respective contributions to $j$ 's input, $W_{k j}$. Consequently, $r_{j}^{(i)}(l)$ is either zero (if $q_{j}^{(i)}(l)<b_{j}$ ) or difference $q_{j}^{(i)}(l)-b_{j}$ otherwise. Similarly, the potential and actual losses of $j$ 's immediate suppliers $k, q_{k}^{(i)}(l)$, and $r_{k}^{(i)}(l)$ are defined identically for each of their own immediate suppliers, and so on. Thus, we can recursively define $q_{j}^{(i)}(l)$ and $r_{j}^{(i)}(l)$ by the following:

$$
\begin{aligned}
& q_{j}^{(i)}(l)=\sum_{k} r_{k}^{(i)}(l) W_{k j}, \\
& r_{j}^{(i)}(l)=\left(q_{j}^{(i)}(l)-b_{j}\right)^{+},
\end{aligned}
$$

where the summation in (4) is taken over $j$ 's immediate suppliers $k$ and $(\cdots)^{+}$is the ramp function which stands for taking the enclosed argument to be zero if it is negative. $q_{j}^{(i)}$ and $r_{j}^{(i)}$ can be interpreted as the input and output losses, respectively, of node $j$ due to $i$. Taken together, (4) and (5) recursively define the loss sustained by a node $j$ by those of its suppliers and ultimately by the loss at $i$.

Consequently, the terminating condition (at the starting node $i$ ) is given by

$$
q_{i}^{(i)}(l)=l .
$$

Figure 1 shows a schematic of a generic network $\mathbf{G}$ and the downstream propagation of loss $l$ from the originating node.

3.2. Breakthrough Scenario. It is difficult to obtain analytical, closed-form solutions to (4) and (5) under general conditions, due to the difficulty in obtaining expressions for the nested use of the ramp function the two equations imply. However, we can obtain a solution for a "breakthrough case" scenario. From the point of view of node $j$, the loss $l$ originating from an upstream node $j$ breaks through if it is strong enough not to be reduced to zero by the resilience of intervening nodes between $i$ and $j$. The latter then receives some fraction of $l$, which subject to its resilience $b_{j}$ may or may not become a real loss $r_{j}^{(i)}$. A weaker $l$ while not achieving breakthrough to $j$, might still be able to do so to one or more of its suppliers upstream. 


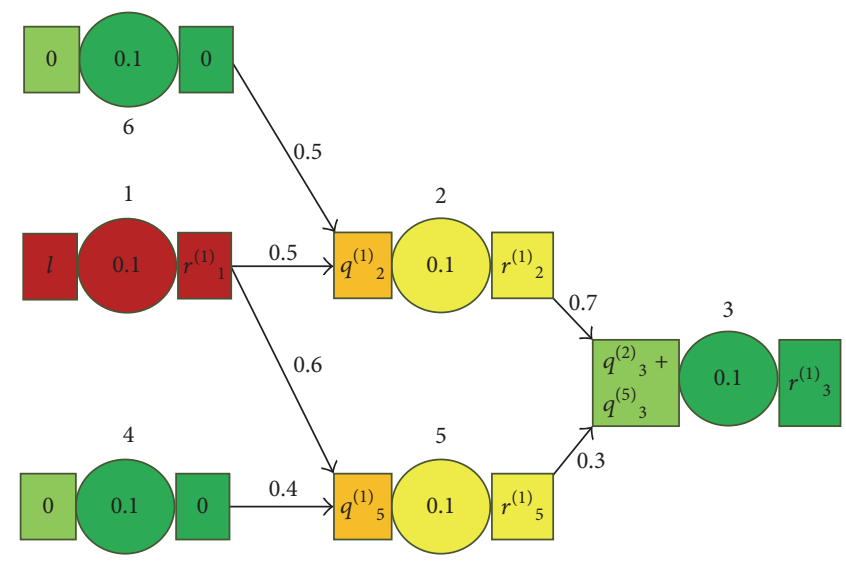

(a) Network

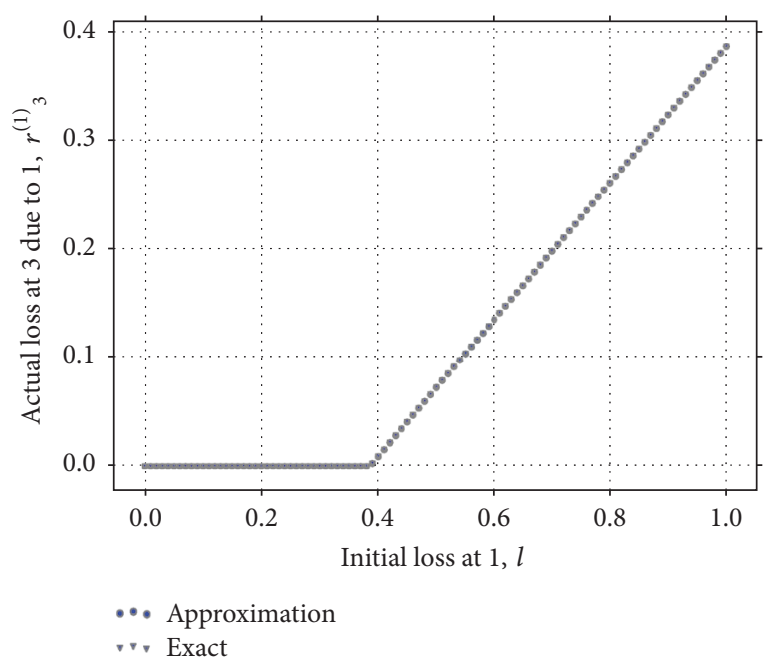

(b) $r_{13}(l)$ versus $l$

Figure 2: (a) Sample network and (b) comparisons of exact and approximate expressions for the loss sustained by node 3 due to a loss at node $1, r_{13}(l)$, against the loss sustained by node $1, l$. The network follows the schema and variables shown as in Figure 1.

This formulation may be useful, as it is often in the interest of a company to determine the potential loss due to a disruption event at a specific supplier upstream, given that supplier's own risk history due to natural (such as earthquakes or cyclones) or anthropogenic causes (such as political or economic instability).

To obtain an analytical solution in the breakthrough regime, we consider the maximum loss, $r_{j}^{*(i)}(l)$, that $j$ can take given a fixed value of $l$. This occurs when the initial loss $l$ at $i$ is strong enough for a portion of it to reach $j$ via the intervening nodes, without getting canceled out by any individual node's resilience threshold. The loss function is thus reduced to a simple difference between $q_{j}^{(i)}(4)$ and its own resilience $b_{j}$. Performing the recursion up to the terminating condition, we can reduce (5) to the following form:

$$
r_{j}^{*(i)}(l)=v_{j}^{(i)} l-\widetilde{b}_{j}^{(i)} .
$$

The breakthrough regime is given by $r_{j}^{*(i)} \geq 0$. Thus, we only need to apply the ramp function to $r_{j}^{*(i)}$ to obtain an expression for $r_{j}^{(i)}$ :

$$
\widetilde{r}_{j}^{(i)}(l)=\left(r_{j}^{*(i)}(l)\right)^{+}=\left(v_{j}^{(i)} l-\widetilde{b}_{j}^{(i)}\right)^{+} .
$$

The first constant, $v_{j}^{(i)}$, can be written in terms of the elements of the powers of the adjacency matrix $[\mathbf{W}]$ :

$$
v_{j}^{(i)}=\sum_{L=1}^{N-1}\left[\mathbf{W}^{L}\right]_{i j},
$$

where we make use of the fact that for a DAG, $\left[\mathbf{W}^{L}\right]_{i j}$ is the sum of the products of the weights along each (unweighted) path of length $L$ leading from $i$ to $j$ (and zero if no such paths are present). $G$ being a DAG also imposes a natural maximum path length of $N-1$. Though the actual maximum path length from $i$ to $j$ may be less than $N-1,\left[\mathbf{W}^{L}\right]_{i j}$ is guaranteed to be zero for path length $L$ above it. $v_{j}^{(i)}$ represents the fraction of inputs to $j$ which ultimately originate from $i$.

For the second constant $\widetilde{b}_{j}^{(i)}$, let $\mathbf{S}_{i j}$ be the set of all nodes which lie on any path from $i$ to $j$, inclusive of $i$ but not $j$. This can be thought of as the set of all suppliers (direct or indirect) of $j$ which takes some input from $i$. Then $\widetilde{b}_{j}^{(i)}$ can be written as

$$
\widetilde{b}_{j}^{(i)}=b_{j}+\sum_{m \in \mathbf{S}_{i j}} \sum_{L=1}^{N-1} b_{m}\left[\mathbf{W}^{L}\right]_{m j},
$$

where $b_{j}$ is $j$ 's resilience threshold and the second term is the average of those of $j$ 's suppliers weighted by their fractional contributions to $j$ 's input. $\widetilde{b}_{j}^{(i)}$ represents the effective resilience threshold of the subnetwork of $\mathbf{G}$ starting from $i$ and ending at $j$.

We compare the results obtained from (8) to exact calculations obtained by explicitly iterating (4) and (5) with (6) as the terminating condition, on a specific network. Figure 2 shows the network, along with results for the exact and approximate calculations for $r_{3}^{(1)}(l)$, the loss sustained by node 3 due to a loss at node 1 (which we take to be the originating node for the loss), as a function of the loss fraction at node $1, l$. The results are identical, showing that the mathematical simplifications stemming from the breakthrough case do not entail loss of accuracy for the single-point-of-origin disruptions we consider. The derivation of an analytical expression for the impacts of simultaneous, multiple-originating-node disruptions upstream of $j$ is beyond the scope of this paper.

3.3. Communicability-Based Impact Measure. In this section, we review the concept of communicability in the network context and apply it to our objective of formulating a measure 
of impact that disruption losses at a node have on another node. Introduced in [16], the communicability between two nodes $i$ and $j$ in a network with unweighted edges is a generalization of the concept of the shortest path between the two and is given by

$$
[\exp (\mathbf{W})]_{i j}=\sum_{m=0}^{+\infty} \frac{\left[\mathbf{W}^{L}\right]_{i j}}{L !},
$$

where $\mathbf{W}$ is the adjacency matrix of the network. It is a weighted sum of the number of walks of length $L$ from $i$ to $j$, where the longer walks are weighted by $1 / L$ !, and can be interpreted as the ease with which information can be transported from $i$ to $j$. A generalization to networks with weighted edges was described in [27]. A further generalization, $f$-communicability, was introduced in [28]. It allows walk weight factors other than $1 / L$ ! to be used, with convergence of the series representation of the function $f$ being the only requirement. In this paper we use a weight factor of $1 / L$; the corresponding $f$-communicability measure becomes

$$
\sum_{L=1}^{+\infty} \frac{\left[\mathbf{W}^{L}\right]_{i j}}{L}=-[\ln (\mathbf{I}-\mathbf{W})]_{i j},
$$

where $\mathbf{I}$ is the identity matrix and $\mathbf{W}$ is the weighed adjacency matrix of the network as defined above. Due to the network being directed and acyclic, the maximum path length is finite, and thus the higher-order $L$ terms in (12) contribute nothing to the sum. Convergence is assured due to each term $\left[\mathbf{W}^{L}\right]_{i j}$ being less than unity. Furthermore, for any $i$ and $j,\left[\mathbf{W}^{L}\right]_{i j}$ will only be nonzero if at least one path of length $L$ exists between the two. We call this measure the logarithmic communicability from $i$ to $j$. Other communicability measures can be used in place of the logarithmic communicability, such as the original (exponential) one, or any function which can be represented as a convergent infinite series. In this work, however, we use the logarithmic form, due to its result, (12), containing the matrix Laplacian of $\mathbf{W},(\mathbf{I}-\mathbf{W})$, which can easily be computed for an arbitrary adjacency matrix. We also note that the right hand side of (12) is positive due to the elements of the matrix logarithm being negative.

A suitable measure of the impact of an initial loss $l$ of $i$ at $j, \mathfrak{\Im}_{j}^{(i)}(l)$, would take into account both the communicability between the two and the actual loss incurred at $j$. Combining (8) and (12) yields the following:

$$
\begin{aligned}
\mathfrak{J}_{j}^{(i)}(l) & =-[\ln (\mathbf{I}-\mathbf{W})]_{i j} \widetilde{r}_{j}^{(i)}(l) \\
& =-[\ln (\mathbf{I}-\mathbf{W})]_{i j}\left(v_{j}^{(i)} l-\widetilde{b}_{j}^{(i)}\right)^{+}
\end{aligned}
$$

with support given by $\left[0,-[\ln (\mathbf{I}-\mathbf{W})]_{i j}\left(v_{j}^{(i)}-\widetilde{b}_{j}^{(i)}\right)\right]$.

3.4. Distribution and Moments of the Impact Measure. Determining the distribution and moments of the impact measure defined in (13) necessitates a choice of density function for the loss, $l$. As it represents a percentage loss in the output of a given node $i$, it must be within the interval $[0,1]$. The beta distribution $B_{\alpha \beta}(l)$ [29] is a suitable choice for its distribution, as this distribution has precisely the required support of $[0,1]$. Furthermore, any distribution defined on this interval can be represented as a weighted sum of beta distributions. With $B(\alpha, \beta)$ denoting the beta function, the density function of $l$ is then given by

$$
B_{\alpha \beta}(l)=\frac{l^{\alpha-1}(1-l)^{\beta-1}}{B(\alpha, \beta)}
$$

for $\alpha$ and $\beta$ both being positive. The two are the shape parameters of the beta distribution, controlling the characteristics of the latter. The distribution's concavity is determined by the values of $\alpha$ and $\beta$ : if at least one of them is less than 1.0, the resulting loss distribution is convex, with one or two maxima appearing at either end of $[0,1]$. If both $\alpha$ and $\beta$ exceed 1.0, the distribution is concave with a single maximum. $\alpha=\beta=1$ corresponds to a uniform loss distribution.

Furthermore, the two control the distribution's skewness: if $\alpha>\beta$, the distribution is left-tailed, and the converse also holds true. $\alpha=\beta$ results in a symmetric distribution. This implies that, for a beta-modeled loss distribution, $\beta>\alpha$ corresponds to a lower probability of sustaining large losses. Finally, $\alpha$ and $\beta$ are related to l's mean, $\bar{l}$, and standard deviation, $\sigma_{l}$, which facilitates analysis of empirical data.

The corresponding distribution of the impact measure, $P\left[\Im_{j}^{(i)}\right]$, can be obtained from (14) by a change of variables and is given by

$$
\begin{aligned}
P\left[\mathfrak{J}_{j}^{(i)}(l)\right]= & \frac{1}{-v_{j}^{(i)}[\ln (\mathbf{I}-\mathbf{W})]_{i j}} \\
& \cdot B_{\alpha \beta}\left[\widetilde{l}_{j}^{(i)}+\frac{\mathfrak{J}_{j}^{(i)}}{-v_{j}^{(i)}[\ln (\mathbf{I}-\mathbf{W})]_{i j}}\right] .
\end{aligned}
$$

Equation (15) is valid in the breakthrough regime given by $l \geq \widetilde{l}_{j}^{(i)}=\widetilde{b}_{j}^{(i)} / v_{j}^{(i)}$.

Denoting the regularized incomplete beta function with parameter $x$ by $I_{x}(\alpha, \beta)$ and making use of the identities

$$
\begin{aligned}
& B(\alpha+k, \beta)=B(\alpha, \beta) \prod_{m=0}^{k-1} \frac{\alpha+m}{\alpha+\beta+m}=\overline{l^{k}} B(\alpha, \beta), \\
& I_{x}(\alpha+k, \beta)=I_{x}(\alpha, \beta)-\sum_{m=0}^{k-1} \frac{x^{\alpha+m}(1-x)^{\beta}}{\overline{l^{m}}(\alpha+m) B(\alpha, \beta)}
\end{aligned}
$$

for integer $k$, it can be shown that, in the breakthrough regime, the $n$th moment of the impact distribution is given by

$$
\begin{aligned}
& \overline{\left(\mathfrak{J}_{j}^{(i)}\right)^{n}} \\
& =\left[v_{j}^{(i)}[\ln (\mathbf{I}-\mathbf{W})]_{i j}\right]^{n} \sum_{k=0}^{n}\left(\begin{array}{l}
n \\
k
\end{array}\right)(-1)^{k}\left(\widetilde{l}_{j}^{(i)}\right)^{n-k} \overline{l^{k}} \\
& \quad \times\left[1+\sum_{m=0}^{k-1} \frac{\left(\widetilde{l}_{j}^{(i)}\right)^{\alpha+m}\left(1-\widetilde{l}_{j}^{(i)}\right)^{\beta}}{(\alpha+m) \overline{l^{m}} B(\alpha, \beta)}-I_{l_{j}^{(i)}}(\alpha, \beta)\right]
\end{aligned}
$$


and is zero otherwise. Here, $\bar{l}$ is the $k$ th moment of the beta distribution $B_{\alpha \beta}(l)$, as given by (16).

The average impact, $\overline{\mathfrak{\Im}}_{j}^{(i)}$, is given by

$$
\begin{aligned}
\overline{\mathfrak{J}_{j}^{(i)}} & =v_{j}^{(i)}[\ln (\mathbf{I}-\mathbf{W})]_{i j}\left\{\widetilde{l}_{j}^{(i)}\left[1-I_{\widetilde{l}_{j}^{(i)}}(\alpha, \beta)\right]\right. \\
& \left.-\bar{l}\left[1+\frac{\left(\widetilde{l}_{j}^{(i)}\right)^{\alpha}\left(1-\widetilde{l}_{j}^{(i)}\right)^{\beta}}{\alpha B(\alpha, \beta)}-I_{\widehat{l}_{j}^{(i)}}(\alpha, \beta)\right]\right\} .
\end{aligned}
$$

In the following two sections, we obtain results for three particular network topologies. First, we examine a perfect $k$-ary tree, where each customer node has exactly $k$ direct suppliers. For this network, we are able to obtain results which are expressed in terms of the branching ratio $k$.

Lastly, we compare results from two networks: one with a random Erdős-Rényi and the other with a scale-free network topology, with a power-law degree distribution [30]. The scale-free network structure has previously been hypothesized to be a characteristic of a robust supply chain network [19].

\section{Results and Discussion}

4.1. Impact for a Perfect $k$-Ary Tree. We consider the impact a supplier node has on a customer in a network with a perfect $k$-ary tree topology with height $h$. In such a topology, all nodes have an indegree $k$ (except for the leaf nodes, which have indegree zero) and an outdegree one, and the leaf nodes are at a distance $h$ away from the root node. Furthermore, we assume that for any node $j$, all its immediate suppliers $\left\{m_{1}, \ldots, m_{k}\right\}$ contribute equally to the input.

In this network, a node $i$ can only have nonzero impact on $j$ only if $j$ is downstream of $i$. In addition, the $i j$ th element of the resulting weighted adjacency matrix, $W_{i j}$, equals $1 / k$ if $i \in\left\{m_{1}, \ldots, m_{k}\right\}$ and zero otherwise. Letting $L_{i j}=h_{i}-h_{j}$ be the unweighted distance between nodes $i$ and $j$ and assuming that the nodes have identical resilience $b_{j}=b$ for all $j$, we can calculate $v_{j}^{(i)}, \widetilde{b}_{j}^{(i)}$, and $\widetilde{l}_{j}^{(i)}$ :

$$
v_{j}^{(i)}=\frac{1}{k^{L_{i j}}},
$$

$$
\begin{aligned}
& \tilde{b}_{j}^{(i)}=b \sum_{m=0}^{L_{i j}} \frac{1}{k^{m}}=b \frac{1-k^{-\left(L_{i j}+1\right)}}{1-k^{-1}}, \\
& \tilde{l}_{j}^{(i)}=b \frac{1-k^{L_{i j}+1}}{1-k} .
\end{aligned}
$$

As the value of $\widetilde{l}_{j}^{(i)}$ must lie between zero and one, (22) imposes an upper bound equaling $(1-k)\left(1-k^{\left(L_{i j}+1\right)}\right)^{-1}$ for the propagation of loss from node $i$ to node $j$. Alternatively, the limit also defines the minimum resilience any node $j$ on the network must have, to prevent losses incurred by supplier $i$ from having an effect on its own output.

Let $\dot{\mathfrak{i}}_{\alpha \beta}^{(2,1)}={\overline{\mathfrak{J}_{j}^{(i)}}}^{(2)} / \overline{\mathfrak{\Im}}_{j}^{(1)}$ be the ratio between the average impact on a node by a supplier for $L_{i j}=2$ and that for $L_{i j}=1$. This is equivalent to the impact ratio between a node's direct supplier's supplier and that direct supplier. We note the wellknown result (e.g., [28]) that, for any network represented by a weighted adjacency matrix $\mathbf{W}$, the $i j$ th element of its $m$ th power, $\left[\mathbf{W}^{m}\right]_{i j}$, is equal to the sum of the products of the weights along each path leading from $i$ to $j$. To put it another way, $\left[\mathbf{W}^{L_{i j}}\right]_{i j}$ is zero if no path of distance $L_{i j}$ from $i$ to $j$ exists. As we are considering a perfect $k$-ary tree configuration, there exists at most only one downstream path of any distance from a node $i$ to another node $j$. The weight of each edge along the path is exactly $1 / k$; thus $\left[\mathbf{W}^{L_{i j}}\right]_{i j}=(k)^{-L_{i j}}$. By (12), the $i j$ th element of the logarithmic communicability $-[\ln (\mathbf{I}-\mathbf{W})]_{i j}$ becomes $L_{i j}^{-1} k^{-L_{i j}}: 1 / k$ for $L_{i j}=1$ and $1 / 2 k^{2}$ for $L_{i j}=2$. Similarly, the effective resilience threshold $\widetilde{b}_{j}^{(i)}$ and the effective loss threshold $\widetilde{l}_{j}^{(i)}$ for the two cases become

$$
\begin{aligned}
& \widetilde{b}_{j}^{(i)(1)}=b \frac{1-k^{-2}}{1-k^{-1}}, \\
& \widetilde{b}_{j}^{(i)(2)}=b \frac{1-k^{-3}}{1-k^{-1}}, \\
& \widetilde{l}_{j}^{(i)(1)}=b \frac{1-k^{2}}{1-k}, \\
& \widetilde{l}_{j}^{(i)(2)}=b \frac{1-k^{3}}{1-k} .
\end{aligned}
$$

Substituting the above equations into (19) yields the following expression for the ratio $\dot{\mathfrak{t}}_{\alpha \beta}$ :

$$
\dot{i}_{\alpha \beta}^{(2,1)}=\frac{1}{2 k^{2}} \frac{\widetilde{l}_{j}^{(i)(2)}\left(1-I_{\widehat{l}_{j}^{(i)(2)}}(\alpha, \beta)\right)-(\alpha /(\alpha+\beta))\left(1+\left(\widetilde{l}_{j}^{(i)(2)}\right)^{\alpha}\left(1-\widetilde{l}_{j}^{(i)(2)}\right)^{\beta} / \alpha B(\alpha, \beta)-I_{\widehat{l}_{j}^{(i)(2)}}(\alpha, \beta)\right)}{\left(1-I_{\widehat{l}_{j}^{(i)(1)}}(\alpha, \beta)\right)-(\alpha /(\alpha+\beta))\left(1+\left(\widetilde{l}_{j}^{(i)(1)}\right)^{\alpha}\left(1-\widetilde{l}_{j}^{(i)(1)}\right)^{\beta} / \alpha B(\alpha, \beta)-I_{\tilde{l}_{j}^{(i)(1)}}(\alpha, \beta)\right)} .
$$

With the restriction given by (22) in mind, we set the node resilience $b$ to be a fraction of the maximum allowed value for $L_{i j}=2: b=\gamma(1-k)\left(1-k^{3}\right)^{-1}$, where $\gamma \in[0,1]$; with this $\tilde{l}_{i j}^{(2)}$ reduces to $\gamma$ and $\widetilde{l}_{j}^{(i)(1)}$ to $\gamma\left(\left(1-k^{2}\right) /\left(1-k^{3}\right)\right) \leq \gamma$.
Using similar arguments we obtain expressions for impact ratios from suppliers of distance other than 1 or 2 from a given node in a $k$-ary tree. In this work we consider the ratios for $L_{i j}=\{2,1\}, L_{i j}=\{3,1\}$, and $L_{i j}=\{4,1\}$. To comply with the restriction given by (22), we define $\gamma$ to be the ratio between $b$ 
and its maximum allowed value for the latter two cases, such that $\widetilde{l}_{j}^{(i)(3)}$ and $\widetilde{l}_{j}^{(i)(4)}$ both reduce to $\gamma$. As the impact measures depend on $\alpha$ and $\beta$, the parameters of the beta distribution, we plot how the ratios vary with both. Figure 3 shows the results for the three ratios previously mentioned.

Figure 3(a) shows that $\dot{i}^{(2,1)}$, the impact ratio on a given customer by its supplier's supplier, as opposed to a direct supplier is substantial across most combinations of $\alpha$ and $\beta$, exceeding 0.45 when the tree is a binary tree $(k=2)$ and the nodes have low resilience $(\gamma=0.1)$; the exception is the region very near the $\beta$ axis, where $\alpha$ is very small compared to $\beta$, corresponding to a beta distribution which is strictly decreasing from $l=0$ to $l=1$, in other words, suppliers with very low-risk of losing production. Here the ratio drops slightly, to approximately $0.41 . \mathfrak{i}^{(2,1)}$ decreases both with increasing the branching ratio $k$ and the node resilience fraction $\gamma$, with the decreases being more prominent in the $\alpha<\beta$ zone. The corresponding loss distribution peaks at $l<0.5$, and the suppliers are more likely to sustain low to moderate losses in production. Even for a binary tree $(k=2)$ and high node resilience $(\gamma=0.9)$, the impact ratio reaches 0.4 in the $\alpha>\beta$ region, corresponding to suppliers likely to sustain moderate to high production losses. We see similar trends for $k=3$ and $k=4$, except that the ratio values decrease, due to the fact that an increasing $k$ increases the number of direct suppliers a customer has and thus decreases the "influence" any single direct supplier has on it.

Thus for $\mathfrak{i}^{(2,1)}$, below the resilience threshold (where any upstream losses are not propagated downstream), decreases in $\gamma$ serve to minimize variations in the impact ratio with $\alpha$ and $\beta$; that is, such decreases minimize the influence of the local conditions of the suppliers. Since at the same time, decreases in $\gamma$ also increase the ratio regardless of $\alpha$ and $\beta$, this implies that the impact of a production loss at direct supplier's supplier (called a Tier 2 supplier in the supply chain field) on a customer will be consistently high in relation to that of a direct supplier (a Tier 1 supplier), regardless of whether the actual chance of that loss happening is low $(\alpha<\beta)$ or high $(\alpha>\beta)$. Increasing $\gamma$ (while keeping below the resilience threshold of $\gamma=1$ ) decreases $\dot{\mathfrak{i}}^{(2,1)}$, with further decreases when $\alpha$ is much smaller than $\beta$.

Figures 3(b) and 3(c), respectively, show the impact ratios of Tier 3 and Tier 4 suppliers to Tier 1 suppliers. We see that the impact ratios are much smaller and are only appreciable for low branching ratio $k$ and resilience fraction $\gamma$, with the maximum values reaching up to 0.24 for $\mathfrak{i}^{(3,1)}$ and 0.11 for $\mathfrak{i}^{(4,1)}$. As with $\mathfrak{i}^{(2,1)}$, increasing $k$ brings reductions in the ratio values, while increasing $\gamma$ corresponds to greater reductions when $\alpha$ is less than $\beta$. Due to the distances of Tier 3 and Tier 4 suppliers from the customer, any production losses they incur either fail to propagate or become attenuated as they propagate to the customer. We see that the attenuation is large for impacts originating from Tier 4 suppliers: $\mathfrak{i}^{(4,1)}$ does not exceed 0.3 when $k=3$, 4 ; even with $k=2$, the ratio does not exceed 0.05 when $\gamma=0.5$ and is practically zero regardless of $\alpha$ and $\beta$ when $\gamma=0.9$. Thus, Tier 4 suppliers (and presumably those of higher tiers) only have noticeable impact when $k$ is minimized (meaning less alternative suppliers for each node in the network) and $\gamma$ is also low (implying that the nodes have less capability to successfully absorb propagating impacts). Meanwhile the loss profile of the supplier (as given by the loss distribution parameters $\alpha$ and $\beta$ ) serves as an additional determinant of whether the impact is high $(\alpha>\beta)$ or low $(\alpha<\beta)$, each corresponding to higher and lower risk of sustaining high losses in production $(l>0.5)$. Tier 3 suppliers have intermediate impact between those of Tier 2 and Tier 4 ones, with the impact more appreciable for lower $k$ and $\gamma$.

While the supply chain surveys previously mentioned in this work $[3,4]$ have data on the distribution of the cumulative losses the surveyed firms suffer in a year as well as data on distance between the originating supplier responsible for the disruption and the surveyed firm, the information reported by the respondents is incomplete, and there is a lack of information from a portion of the respondents. Nevertheless, 2017 survey data on the proportion of firms which reported predominant disruptions from Tier 1, Tier 2 , and Tier 3 and upstream suppliers at $44 \%, 24 \%$, and $10 \%$ supplier disruptions, respectively, may be suggestive. Disruptions from Tier 1 suppliers have a higher impact on focal companies compared to those from Tier 2 and below. With comparison with Figure 3(a), the ratios between the different tiers suggest the prevalence of a supply chain network structure with low redundancy and low effective resilience. However, more data are needed; $22 \%$ of companies surveyed did not trace the sources of disruption that year, and the survey did not ask respondents to provide disruption loss data segregated by supplier tier, suggesting an avenue for further empirical research.

4.2. Impact for Two Networks. We also consider the impact on downstream nodes in two networks: one a scale-free DAG generated by the algorithm proposed in [31] and the other an Erdös-Rényi DAG [32], each with $N=250$ nodes. The two networks were generated such that their average indegrees (the average number of Tier 1 suppliers per node) are close to each other. Each node was given a resilience threshold $b_{i}$ drawn uniformly from the interval $[0.01,0.03]$, while the (unnormalized) weight between adjacent nodes, which is taken to be how much a node supplies to a direct customer, was taken from a uniform distribution between 100.0 and 900.0. Unlike the $k$-ary tree case, the nodes in the generated network are not identical. Figure 4 shows sample distributions for $l$ at node $i$ and the resulting impact two nodes downstream $(j)$ in both networks, following (15).

In our sample distributions, we see zero impact on $j$ from $i$ in the scale-free network throughout the range of possible values of the initial loss $l$, which indicates that any loss does not result in an actual loss downstream. In the E-R network's sample distribution, breakthrough occurs at a low threshold $(l=0.23)$.

Due to the nodes and edges not being identical even within each network, the results we obtain from the sample distributions, while interesting, are not sufficient. However, distributions we obtained across supplier nodes (i.e., all Tier 1, Tier 2, or Tier 3 suppliers) in each network of the average impact given by (19) bear them out: the per-tier distributions 

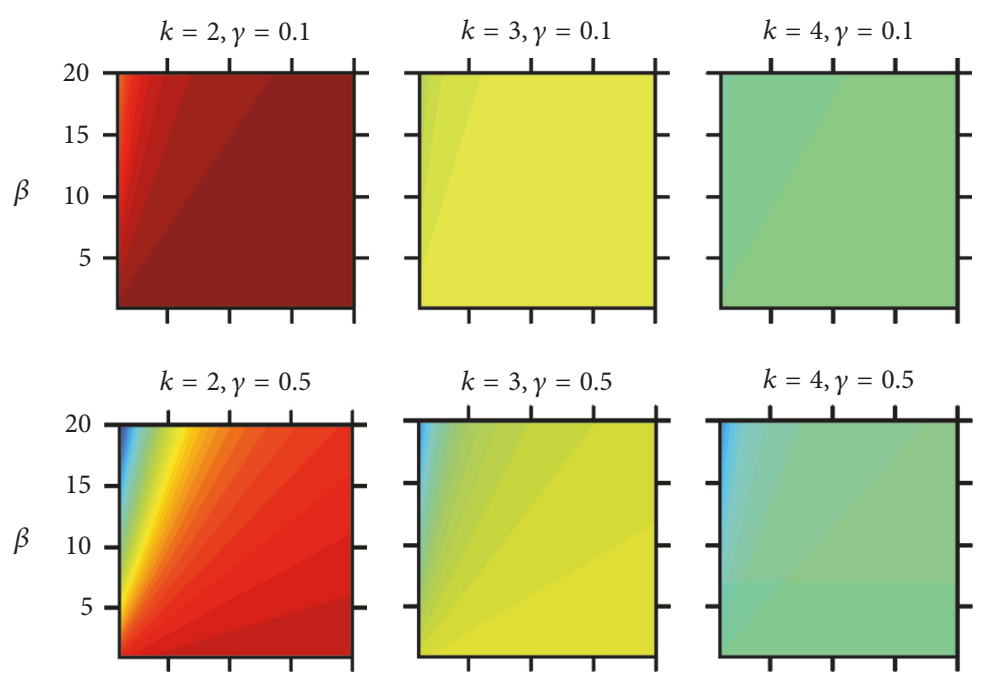

0.4552
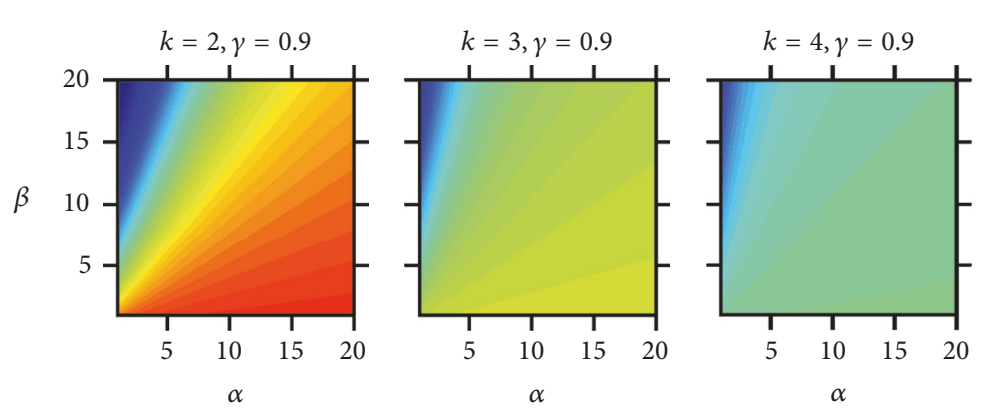

(a) $\mathfrak{i}^{(2,1)}$
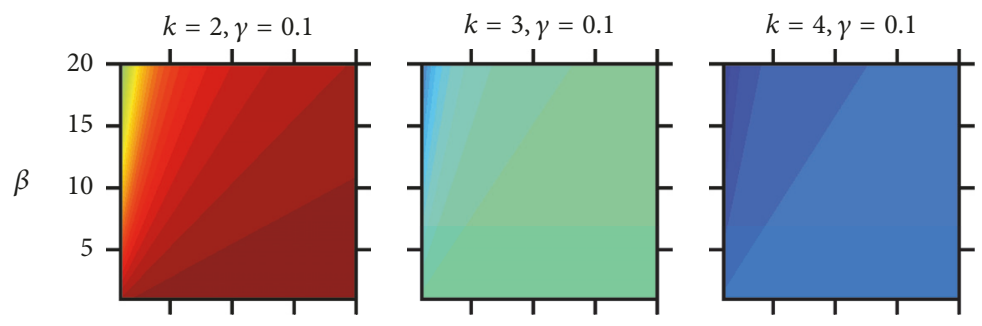

0.4079
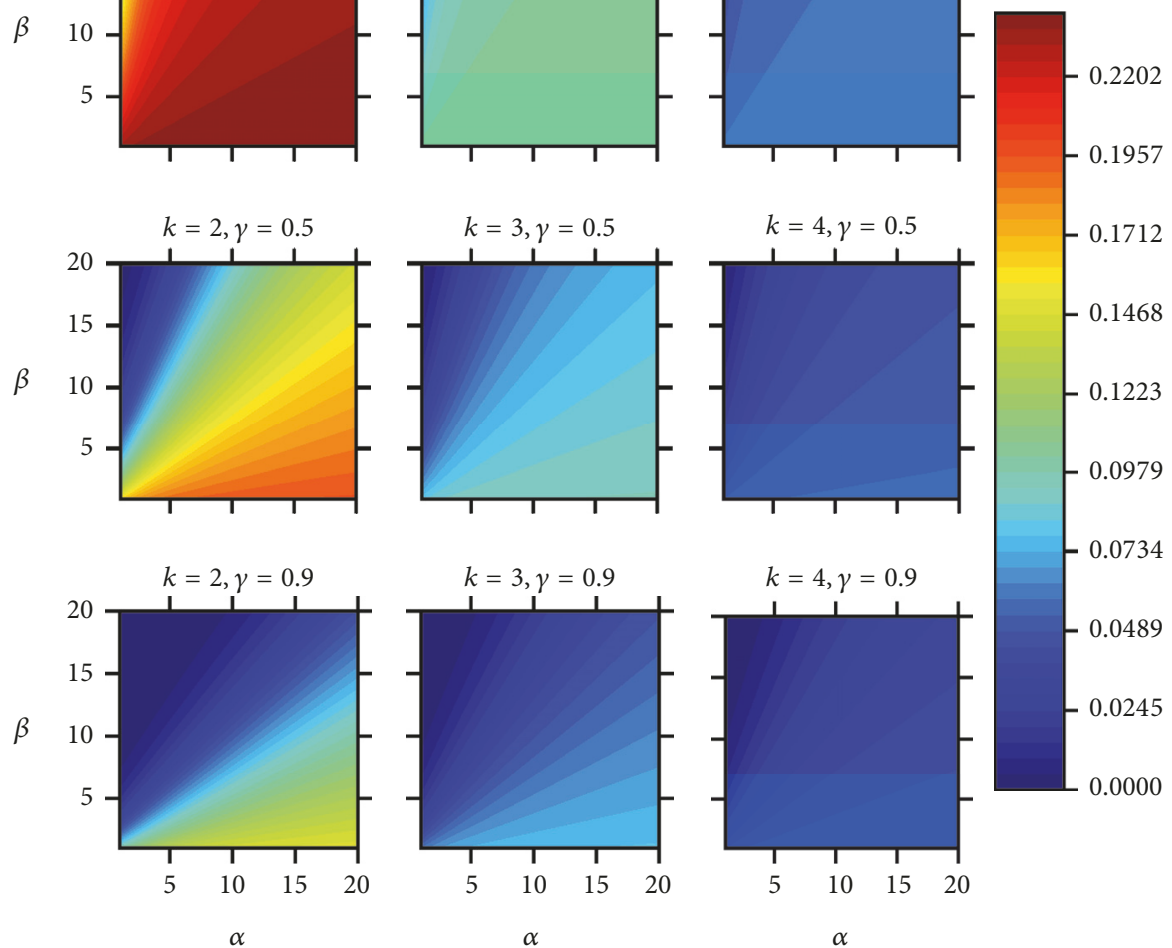

(b) $\mathfrak{i}^{(3,1)}$

FIgURE 3: Continued. 

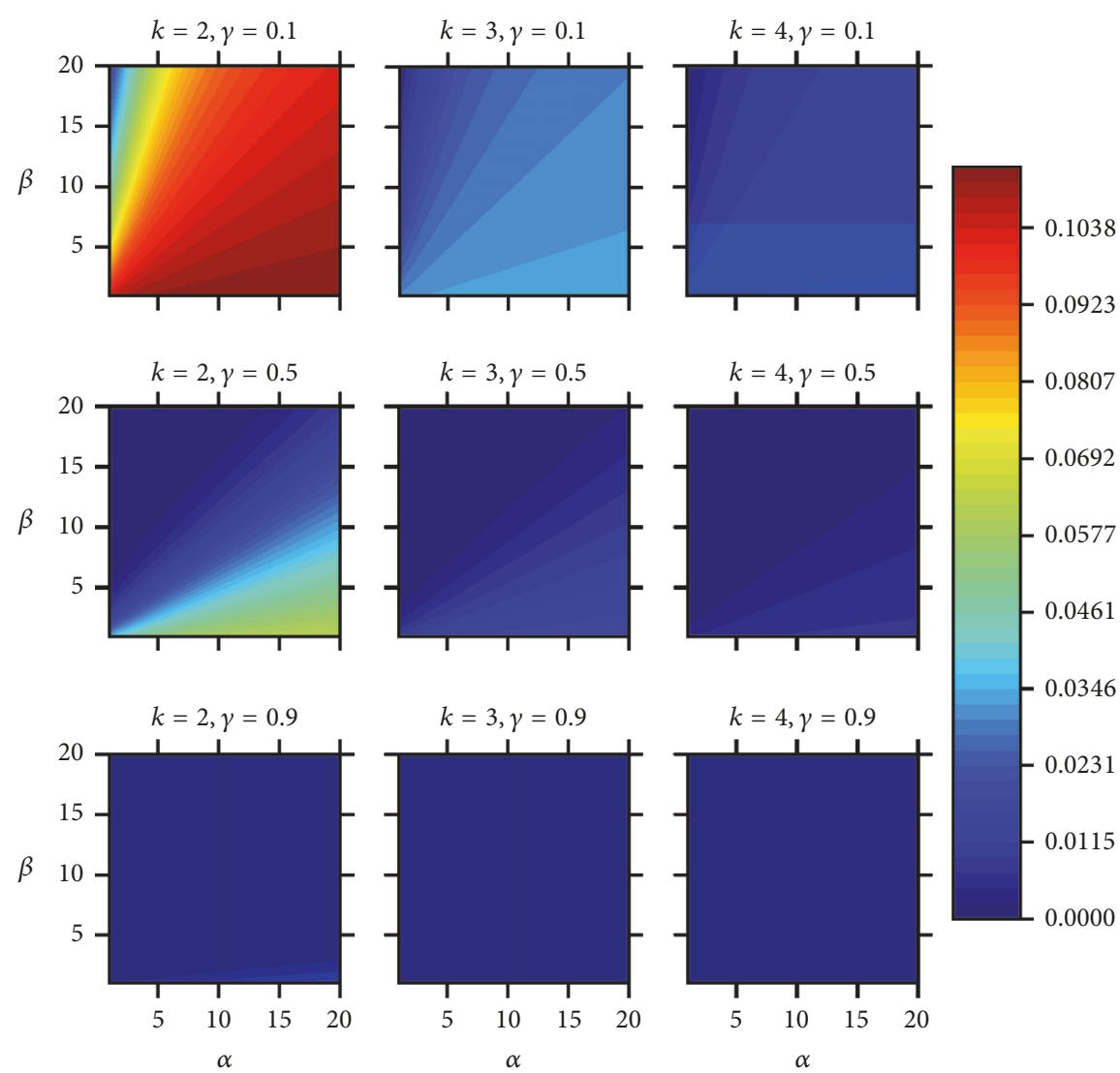

(c) $\mathfrak{i}^{(4,1)}$

FIGURE 3: Impact ratios for varying supplier distance, $L_{i j}$, beta distribution parameters $\alpha$ and $\beta$, branching ratio $k$, and resilience fraction $\gamma$.

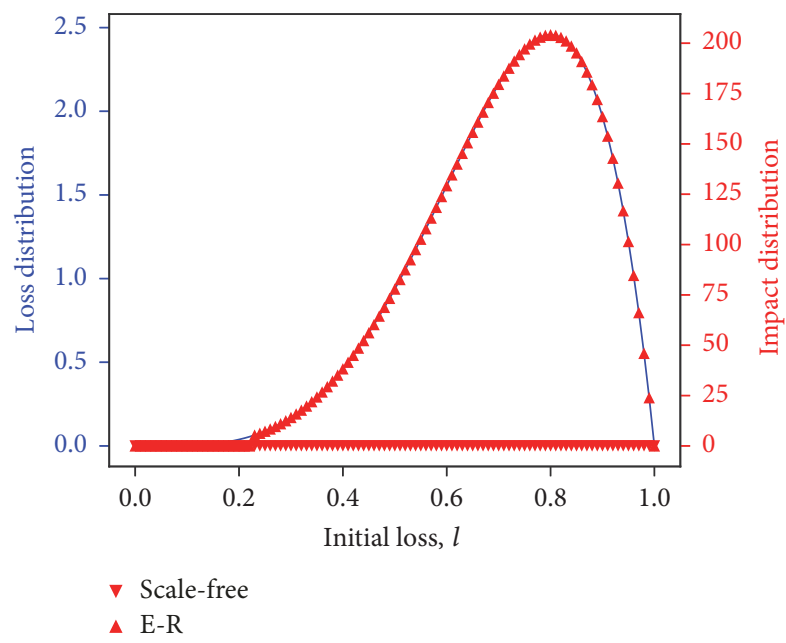

FIGURE 4: Initial loss and impact distributions for two directed acyclic graphs: a scale-free network ("scale-free") and an ErdösRényi network ("E-R"), in which the loss $l$ originates at node $i$ two nodes upstream of node $j$ and obeys a beta distribution $(\alpha=5.0$, $\beta=2.0)$.

in the E-R network are wider than their counterparts in the scale-free network and thus have higher median impact for the same initial loss distribution (see Figure 5).
The results support previous arguments for the robustness of supply chain networks with a scale-free network topology [19]; Figure 5 shows that the two networks, which are otherwise very similar in size, average indegree, node and edge characteristics, and loss profiles, nevertheless have different behaviors, with the scale-free network featuring smaller impacts from suppliers regardless of tier.

\section{Conclusion}

In this work, we have formulated a network-based measure of the impact of a disruption at one part of a supply network on the production of another part. We did this by tracking the propagation of an initial production loss sustained by a node downstream to another node, across intervening nodes. The measure takes into account the risk profile of the originating node, the structure of the supply network, and the resilience capabilities of the components of the network. By an analogous process we also derived the loss sustained by any node in the network resulting from an upstream initial loss which has propagated downstream.

The results of this study offer several insights into the relationship between the impact of disruption losses a supplier has on a downstream customer, the distance between the two within the network, and the degree of resilience to loss 


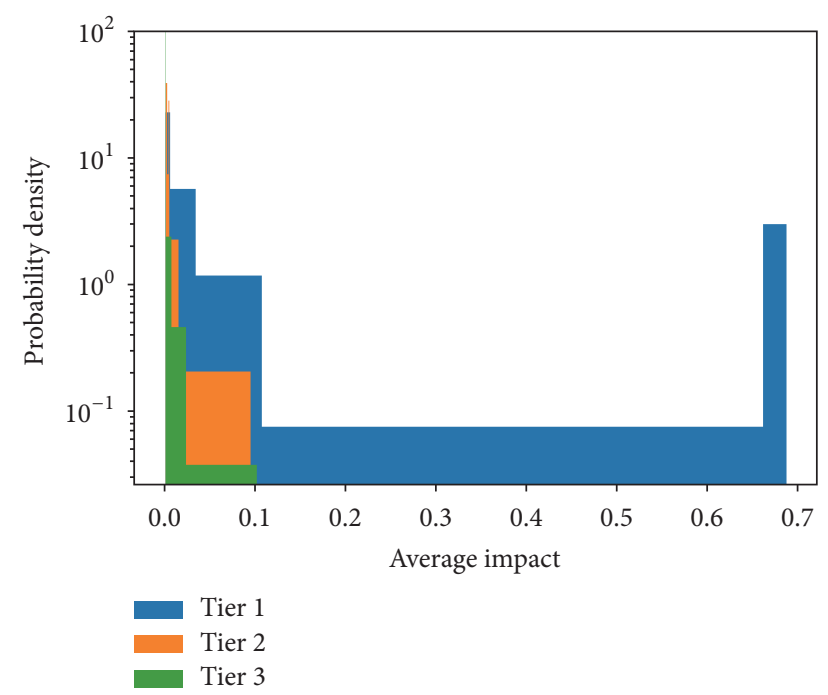

(a) Scale-free network

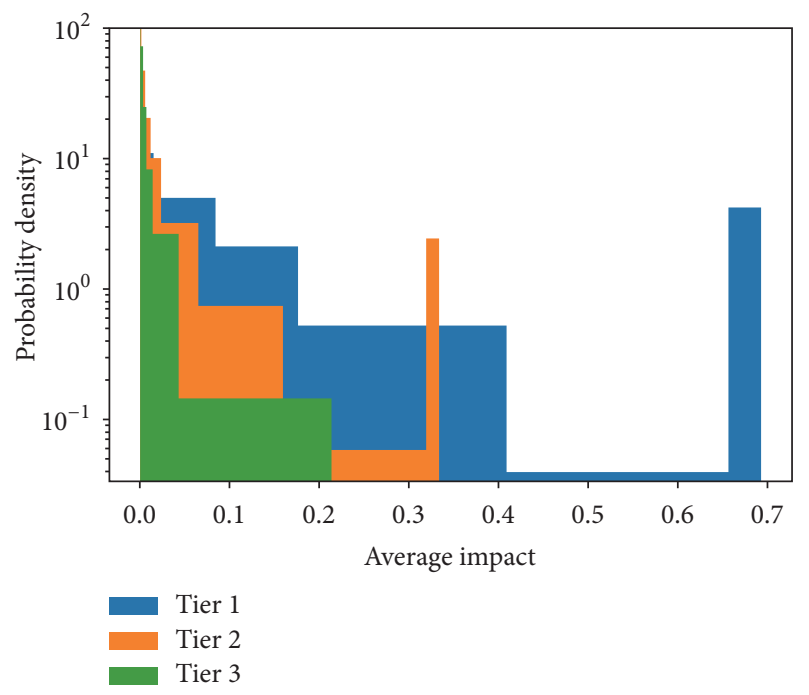

(b) Erdős-Rényi network

FIgURE 5: Histograms of the average impact $\overline{\mathfrak{\Im}_{j}^{(i)}}$, across all possible node pairs $(i, j)$ in which $i$ is a Tier 1/Tier 2/Tier 3 supplier of $j$ and the initial loss $l$ is beta-distributed $(\alpha=5.0, \beta=2.0)$. Medians: Tier 1: 0.0002 (scale-free), 0.0817 (E-R); Tier 2: 0.0 (scale-free), 0.0056 (E-R); Tier 3: 0.0 (scale-free), 0.0004 (E-R).

intervening nodes have. Distance, or equivalently the presence of intervening nodes between supplier and customer, reduces the production loss the customer can sustain if the intervening nodes have some degree of resilience. This loss can be further reduced and the influence of the supplier's local loss profile can be attenuated, if the intervening nodes have higher resilience from potential losses. This is desirable from a customer-oriented point of view, with propagated losses both minimal and independent of suppliers' circumstances. On the other extreme, with minimal resilience and minimal number of intervening nodes, the losses a customer can sustain are large. The relative impacts of suppliers of different distances to a customer are also determined by the structure of the subnetwork involving the suppliers and the customer, which controls both the relative contribution a supplier has on the customer and the effective resilience of the part of the network lying between the two.

The overall structure of the supply chain network also plays a role in determining whether an upstream loss is able to break through to downstream customers; our results show the robustness of a scale-free structured network compared to a random network of similar characteristics. The average impacts from upstream supplier losses in such networks are lower than their random-network counterparts.

The analytic model proposed in this work can be applied to measure the robustness of a focal company's supply chain network, given the disruption profile of supplier nodes and the network structure of the entire supply chain. This is by identifying the suppliers that could potentially have the most impact upon sustaining disruption losses and determining changes in impact with different network structures, or alternative supplier firms with different risk profiles. Thus, this work clearly illustrates the need for supply chain managers to build risk profiles of each component of a supply chain network and inform relevant components in developing corresponding mitigation strategies for failure events.

The proposed method enables a focal company to estimate the impact of losses from different supplier tiers. Furthermore, this work suggests the significance of including in future surveys the distribution of supplier tiers for a particular amount of loss sustained. Knowledge of this allows one to obtain the contributions of each supplier tier to one's own loss profile.

\section{Conflicts of Interest}

The authors declare that they have no conflicts of interest.

\section{Acknowledgments}

The authors are deeply indebted to and gratefully acknowledge Dr. Christopher Monterola for helpful and useful discussions. This research was partially supported by Singapore A*STAR Science and Engineering Research Council (Grant no. 1121790043) (JFBV, XF, and RSMG) and Ministry of Education, Singapore (Grant no. MOE2016-T2-1-119) (Gaoxi Xiao).

\section{References}

[1] M. Haraguchi and U. Lall, "Flood risks and impacts: A case study of Thailand's floods in 2011 and research questions for supply chain decision making," International Journal of Disaster Risk Reduction, vol. 14, pp. 256-272, 2015.

[2] Y. Saito, M. Nirei, and V. Carvalho, "Supply chain disruptions: Evidence from great east japan earthquake," in Society for Economic Dynamics, Supply chain disruptions, Evidence from great east japan earthquake, 2014 Meeting Papers 595.

[3] P. Alcantara, G. Riglietti, and L. Aguada, "Bci supply chain resilience report," Tech. Rep., Business Continuity Institute, 2017. 
[4] P. Alcantara and G. Riglietti, "Bci supply chain resilience report," Tech. Rep., Business Continuity Institute, 2016.

[5] A. Halldorsson, H. Kotzab, J. H. Mikkola, and T. Skjøtt-Larsen, "Complementary theories to supply chain management," Supply Chain Management Review, vol. 12, no. 4, pp. 284-296, 2007.

[6] A. G. Haldane and R. M. May, "Systemic risk in banking ecosystems," Nature, vol. 469, no. 7330, pp. 351-355, 2011.

[7] A. Laszka, B. Johnson, J. Grossklags, and M. Felegyhazi, "Estimating systematic risk in real-world networks," Lecture Notes in Computer Science (including subseries Lecture Notes in Artificial Intelligence and Lecture Notes in Bioinformatics): Preface, vol. 8437, pp. 417-435, 2014.

[8] L. Shabnam, F. Haque, M. Bhuiyan, and A. Krishna, "Risk measure propagation through organisational network," in Proceedings of the 38th Annual IEEE Computer Software and Applications Conference Workshops, COMPSACW 2014, pp. 217222, Sweden, July 2014.

[9] F. Blöchl, F. J. Theis, F. Vega-Redondo, and E. O. Fisher, "Vertex centralities in input-output networks reveal the structure of modern economies," Physical Review E: Statistical, Nonlinear, and Soft Matter Physics, vol. 83, no. 4, Article ID 046127, 2011.

[10] S. S. Chopra and V. Khanna, "Interconnectedness and interdependencies of critical infrastructures in the US economy: Implications for resilience," Physica A: Statistical Mechanics and its Applications, vol. 436, pp. 865-877, 2015.

[11] D. Acemoglu, A. Ozdaglar, and A. Tahbaz-Salehi, "Networks, Shocks, and Systemic Risk," National Bureau of Economic Research w20931, 2015.

[12] S. V. Buldyrev, R. Parshani, G. Paul, H. E. Stanley, and S. Havlin, "Catastrophic cascade of failures in interdependent networks," Nature, vol. 464, no. 7291, pp. 1025-1028, 2010.

[13] A. Vespignani, "Complex networks: The fragility of interdependency," Nature, vol. 464, no. 7291, pp. 984-985, 2010.

[14] I. Kilubi and H.-D. Haasis, "Supply chain risk management enablers-A framework development through systematic review of the literature from 2000 to 2015," International Journal of Business Science and Applied Management, vol. 10, no. 1, pp. 3554, 2015.

[15] W. Ho, T. Zheng, H. Yildiz, and S. Talluri, "Supply chain risk management: A literature review," International Journal of Production Research, vol. 53, no. 16, pp. 5031-5069, 2015.

[16] E. Estrada and N. Hatano, "Communicability in complex networks," Physical Review E: Statistical, Nonlinear, and Soft Matter Physics, vol. 77, no. 3, Article ID 036111, 2008.

[17] M. S. Sodhi, B.-G. Son, and C. S. Tang, "Researchers' perspectives on supply chain risk management," Production Engineering Research and Development, vol. 21, no. 1, pp. 1-13, 2012.

[18] C. S. Tang, "Perspectives in supply chain risk management," International Journal of Production Economics, vol. 103, no. 2, pp. 451-488, 2006.

[19] E. J. S. Hearnshaw and M. M. J. Wilson, "A complex network approach to supply chain network theory," International Journal of Operations \& Production Management, vol. 33, no. 4, pp. 442469, 2013.

[20] D. L. Olson and S. R. Swenseth, "Trade-offs in Supply Chain System Risk Mitigation," Systems Research and Behavioral Science, vol. 31, no. 4, pp. 565-579, 2014.

[21] A. Nagurney and Q. Qiang, "Fragile networks: identifying vulnerabilities and synergies in an uncertain age," International Transactions in Operational Research, vol. 19, no. 1-2, pp. 123160, 2012.
[22] M. D. Garvey, S. Carnovale, and S. Yeniyurt, "An analytical framework for supply network risk propagation: a Bayesian network approach," European Journal of Operational Research, vol. 243, no. 2, pp. 618-627, 2015.

[23] A. Käki, A. Salo, and S. Talluri, "Disruptions in Supply Networks: A Probabilistic Risk Assessment Approach," Journal of Business Logistics, vol. 36, no. 3, pp. 273-287, 2015.

[24] H. Yildiz, J. Yoon, S. Talluri, and W. Ho, "Reliable Supply Chain Network Design,” Decision Sciences, vol. 47, no. 4, pp. 661-698, 2016.

[25] H.-Y. Xu, X. Fu, L. Ponnambalam, A. Namatame, X. F. Yin, and R. S. M. Goh, "A model to evaluate risk propagation considering effect of dynamic risk information sharing and multi-sourcing in supply chain networks," in Proceedings of the IEEE International Conference on Industrial Engineering and Engineering Management, IEEM 2015, pp. 1593-1597, Singapore, December 2015.

[26] M. E. J. Newman, Networks: An Introduction, Oxford University Press, Oxford, UK, 2010.

[27] J. J. Crofts and D. J. Higham, "A weighted communicability measure applied to complex brain networks," Journal of the Royal Society Interface, vol. 6, no. 33, pp. 411-414, 2009.

[28] E. Estrada and D. J. Higham, "Network properties revealed through matrix functions," SIAM Review, vol. 52, no. 4, pp. 696$714,2010$.

[29] A. Gupta and S. Nadarajah, "Mathematical properties of the beta distribution," in Handbook of Beta Distribution and Its Applications, A. K. Gupta and S. Nadarajah, Eds., vol. 174, Chapter 2, pp. 33-53, CRC Press, Dekker, New York, NY, USA, 2004.

[30] A.-L. Barabási and R. Albert, "Emergence of scaling in random networks," American Association for the Advancement of Science: Science, vol. 286, no. 5439, pp. 509-512, 1999.

[31] B. Bollobás, C. Borgs, J. Chayes, and O. Riordan, "Directed scale-free graphs," in Proceedings of the Fourteenth Annual ACM-SIAM Symposium on Discrete Algorithms, SODA '03, Society for Industrial and Applied Mathematics, pp. 132-139, ACM, New York, NY, USA, 2003.

[32] P. Erdös and A. Rényi, "On random graphs," Publicationes Mathematicae, vol. 6, pp. 290-297, 1959. 


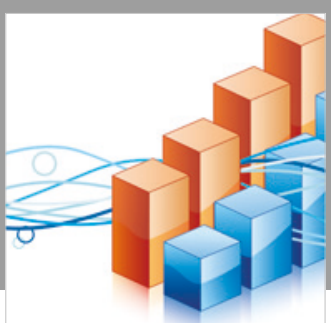

Advances in

Operations Research

\section{-n-m}
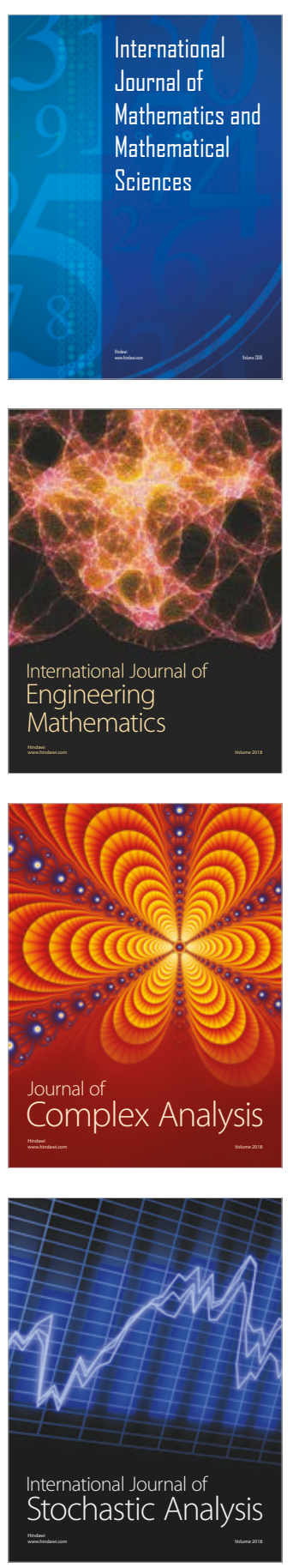
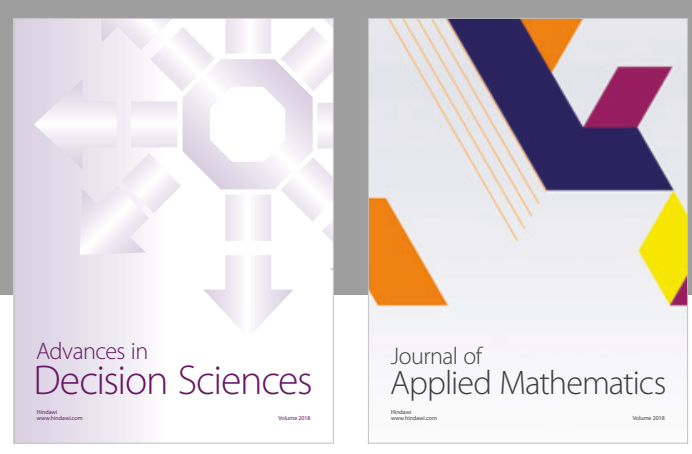

Journal of

Applied Mathematics
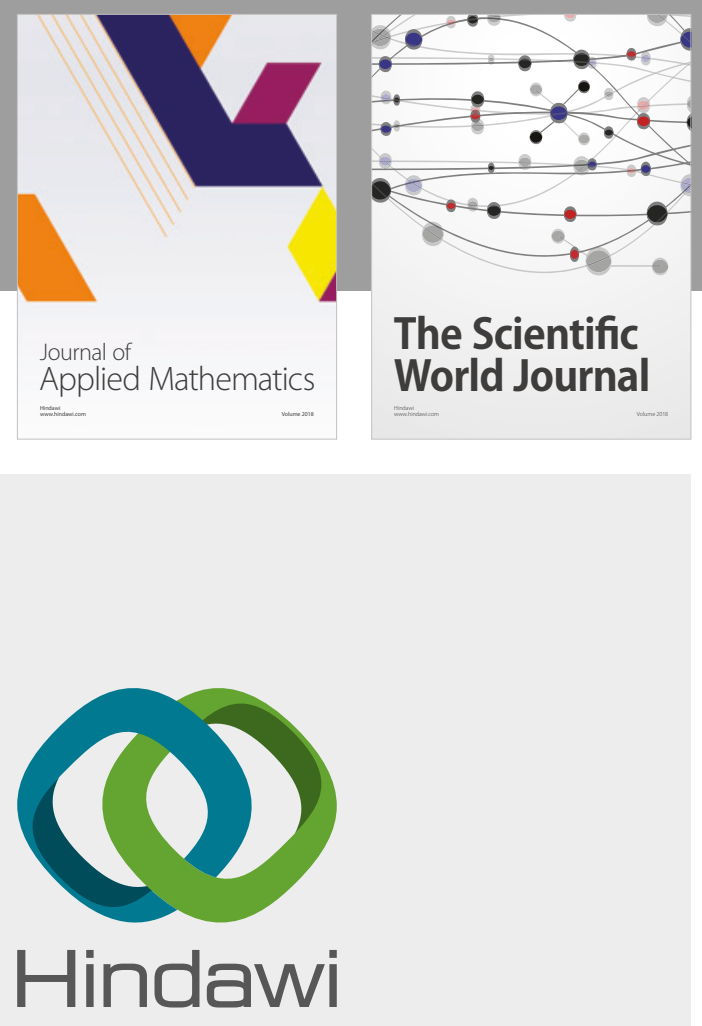

Submit your manuscripts at

www.hindawi.com

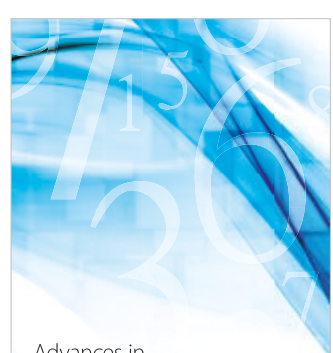

Advances in
Numerical Analysis
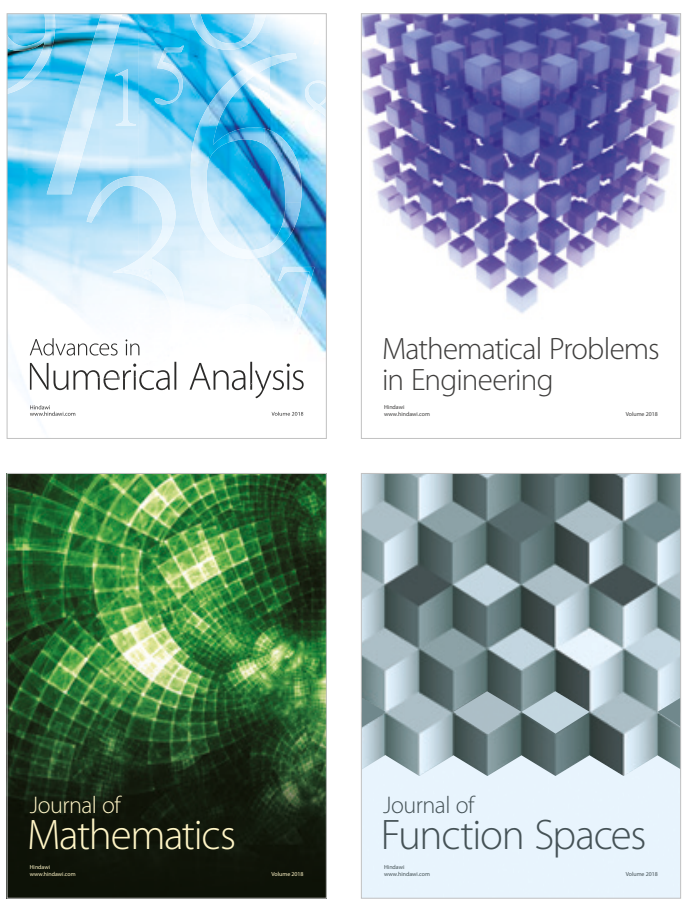

Mathematical Problems in Engineering

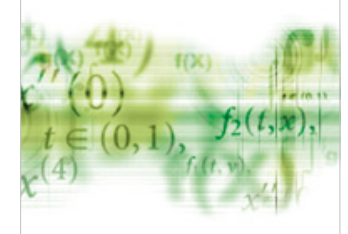

International Journal of

Differential Equations

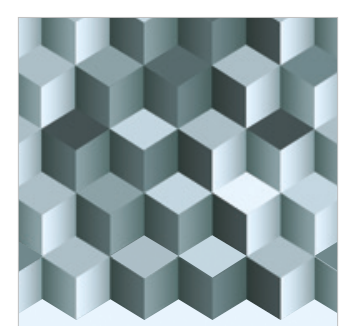

Journal of

Function Spaces
The Scientific

World Journal

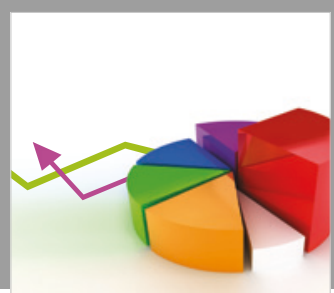

Journal of

Probability and Statistics
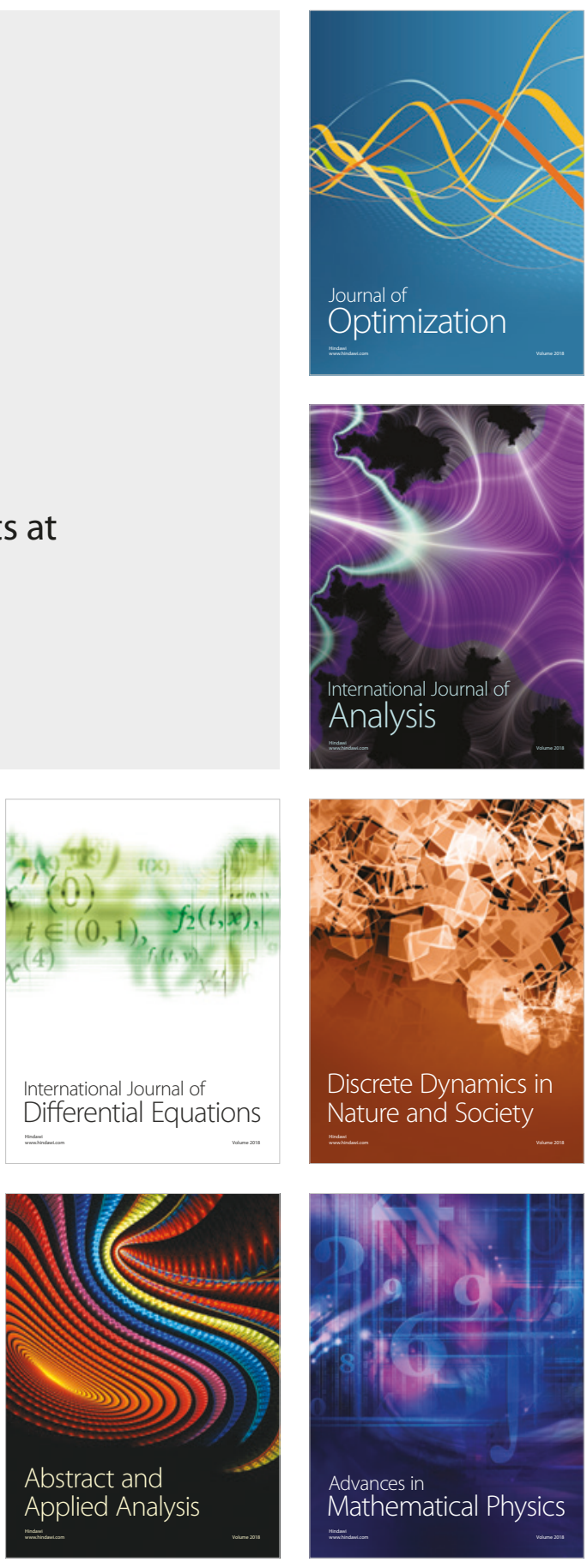